\title{
ON SOME SOLUTIONS TO THE KLEIN-GORDON EQUATION RELATED TO AN INTEGRAL OF SONINE
}

BY

\author{
STUART NELSON
}

\begin{abstract}
An integral due to Sonine is used to obtain an expansion for special solutions $W(x, t)$ of the Klein-Gordon equation. This expansion is used to estimate the $L_{p}$ norms $\|W(\cdot, t)\|_{p}$ as $t \rightarrow \infty$. These estimates yield results on the time decay of a fairly wide class of solutions to the Klein-Gordon equation.
\end{abstract}

1. Introduction. We intend to study the functions $W_{t, a}(x), x \in R^{n}$ whose Fourier transforms are $\hat{W}_{t, a}(y)=\left(1+y^{2}\right)^{-a} \exp \left[-i t\left(1+y^{2}\right)^{1 / 2}\right]$. If $a>n / 4$, the existence of such functions $W_{t, a} \in L_{2}\left(R^{n}\right)$ is assured by Plancherel's theorem. Indeed, $W_{t, a}$ is the $L_{2}$ limit of $W_{t, a, R}$ as $R \rightarrow \infty$ where

$$
W_{t, a, R}(\boldsymbol{x})=(2 \pi)^{-n / 2} \int_{|y|<R} e^{i x \cdot y} \frac{\exp \left[-i t\left(1+y^{2}\right)^{1 / 2}\right]}{\left(1+y^{2}\right)^{a}} d y_{1} \cdots d y_{n}
$$

We are interested in the behavior of the functions $W_{t, a}(x)$ for large values of $t$. Our main objective is to obtain estimates on the $L_{p}\left(R^{n}\right)$ norms $\left\|W_{t, a}\right\|_{p}$ as $t \rightarrow \infty$.

This study of the functions $W_{t, a}$ was begun by A. R. Brodsky in [3] where he showed that many solutions of the Klein-Gordon equation satisfy an inequality of the form

$$
\|u(\cdot, t)\|_{\infty} \leqq C_{u, a}\left\|W_{t, a}\right\|_{\infty} .
$$

Here the constant is determined by the Cauchy data $u(x, 0), u_{t}(x, 0)$ which must satisfy certain regularity assumptions. (The amount of regularity required depends on the choice of $a$ and increases as $a$ is increased.) Brodsky proved that for certain values of $a,\left\|W_{t, a}\right\|_{\infty}=O\left(t^{(1-n) / 2}\right)$ as $t \rightarrow \infty$. This paper shows that for $a \geqq(n+2) / 4,\left\|W_{t, a}\right\|_{\infty}=O\left(t^{-n / 2}\right)$ so that by (1.2) solutions $u(x, t)$ decay like $t^{-n / 2}$. Such $t^{-n / 2}$ bounds on solutions of the Klein-Gordon equation have already been obtained by Segal [6, pp. 95-98] and also follow from Littman [9]. The advantage of the present approach is that it requires fewer assumptions on the Cauchy data.

Much of the motivation for this study has come from I. E. Segal. He has posed many fruitful questions and in [7] has made use of Corollaries 5.1 and 5.2 to deal

Received by the editors December 19, 1969 and, in revised form, February 6, 1970.

AMS 1968 subject classifications. Primary 3516, 3537, 3552; Secondary 3325, 4240, 8135, 8335.

Key words and phrases. Klein-Gordon equation, Cauchy problem, asymptotic behavior, estimate of $L_{p}$ norms, Fourier transform, Sonine's discontinuous integral, Bessel functions.

Copyright (C) 1971, American Mathematical Society 
with situations where the Cauchy data is not regular enough for the $t^{-n / 2}$ bounds to be applicable. Another application of results from this paper will be given in [5].

This investigation owes its beginning to N. Levinson's suggestion that bounds on $\left\|W_{t, a}\right\|_{\infty}$ be obtained by using the method of stationary phase to estimate the integral in equation (2.3) below. This was carried out in [4] with his guidance. The present paper includes most of the results of [4] but is based on a different method whereby the integral in (2.3) is approximated by an integral due to Sonine. This is done in $\S 2$ and leads to an expansion for $W_{t, a}$, the terms of which are analyzed in $\S 3$. In $\S 4$ we estimate the remainder term of this expansion and in $\$ 5$ we arrive at some conclusions about $W_{t, a}$.

2. An expansion for $W_{t, a}(x)$. Suppose $G \in L_{1}\left(R^{n}\right)$ is spherically symmetric so that $G(x)$ depends only on $x=(x \cdot x)^{1 / 2}$. Then

$$
F(\boldsymbol{r})=(2 \pi)^{-n / 2} \int_{R^{n}} e^{i x \cdot r} G(\boldsymbol{x}) d x_{1} \cdots d x_{n}
$$

depends only on $r=(\boldsymbol{r} \cdot \boldsymbol{r})^{1 / 2}$. Indeed, after switching to spherical coordinates in (2.1) and doing the angular part of the integration (see Bochner [2, Theorem 40]) one is left with

$$
F(\boldsymbol{r})=r^{-\mu} \int_{0}^{\infty} \rho^{\mu+1} J_{u}(r \rho) G(\rho, 0, \ldots, 0) d \rho
$$

where $\mu=n / 2-1$ and $J_{\mu}$ is a Bessel function of the first kind.

Applying this result to (1.1) we have

$$
W_{t, a, R}(\boldsymbol{r})=r^{-\mu} \int_{0}^{R} \rho^{\mu+1} J_{\mu}(r \rho)\left(1+\rho^{2}\right)^{-a} \exp \left[-i t\left(1+\rho^{2}\right)^{1 / 2}\right] d \rho .
$$

If $a>(n+1) / 4$ this integral is absolutely integrable over $0 \leqq \rho<\infty$ so that

$$
W_{t, a}(\boldsymbol{r})=\int_{0}^{\infty} \frac{x^{\mu+1}}{r^{\mu}} J_{\mu}(r x) \frac{\exp \left[-i t\left(1+x^{2}\right)^{1 / 2}\right]}{\left(1+x^{2}\right)^{a}} d x .
$$

The integral (2.3) will be approximated by means of an integral due to Sonine:

$$
\int_{0}^{\infty} \frac{x^{\mu+1}}{r^{\mu}} J_{\mu}(r x) \frac{K_{v}\left(\tau\left(1+x^{2}\right)^{1 / 2}\right)}{\left(1+x^{2}\right)^{v / 2}} d x=\tau^{-v}\left(\left(r^{2}+\tau^{2}\right)^{1 / 2}\right)^{\nu-\mu-1} K_{v-\mu-1}\left(\left(r^{2}+\tau^{2}\right)^{1 / 2}\right)
$$

where $K_{v}$ is a modified Bessel function of the second kind. For $\tau>0$ equation (2.4) is established in $\$ 13.47$ of Watson [8] and by analytic continuation (2.4) holds for $\operatorname{Re} \tau>0$. If $\tau=i t, t>0$, the factor $K_{v}\left(\tau\left(1+x^{2}\right)^{1 / 2}\right)$ no longer decreases exponentially and in order that the integral be absolutely convergent one must assume $\nu>\mu+1$. With this assumption both sides of (2.4) are continuous for $\operatorname{Re} \tau \geqq 0, \tau \neq 0$, and hence the fact that (2.4) holds for $\operatorname{Re} \tau>0$ implies that it holds for $\tau=i t, t>0$. Rewriting (2.4) for this case we have

$$
\int_{0}^{\infty} \frac{x^{\mu+1}}{r^{\mu}} J_{\mu}(r x) \frac{K_{v}\left(i t\left(1+x^{2}\right)^{1 / 2}\right)}{\left(i t\left(1+x^{2}\right)^{1 / 2}\right)^{v}} d x=(i t)^{-2 v} u^{v-\mu-1} K_{\nu-\mu-1}(u)
$$

where $u=u(r, t)=\left(r^{2}-t^{2}\right)^{1 / 2}$ is positive when $r>t$ and satisfies $u=|u| e^{i \pi / 2}$ when $r<t$. 
To use (2.5) to approximate (2.3) we need

LEMMA 2.1. For every real number a there exist unique constants $C_{0}(a)$, $C_{1}(a), \ldots, C_{N}(a), \ldots$ such that the functions $B_{N, a}(z)$ defined by

$$
\frac{e^{-z}}{z^{2 a}}=\sum_{k=0}^{N} C_{k}(a) \frac{K_{k+2 a-1 i 2}(z)}{z^{k+2 a-1 / 2}}+\frac{e^{-z} B_{N, a}(z)}{z^{N+2 a+1}}
$$

satisfy

$$
\infty>\left|B_{N, a}\right|_{\varepsilon} \equiv \sup \left\{\left|B_{N, a}(z)\right|:|z| \geqq \varepsilon,|\arg z| \leqq \pi / 2\right\}
$$

for every $\varepsilon>0$.

Proof. From the asymptotic expansion

$$
\begin{aligned}
K_{v}(z)=\left(\frac{\pi}{2 z}\right)^{1 / 2} e^{-z}\{1 & +\frac{4 v^{2}-1}{1 ! 8 z}+\cdots \\
& \left.+\frac{\left(4 \nu^{2}-1\right) \cdots\left(4 \nu^{2}-(2 N-1)^{2}\right)}{N !(8 z)^{N}}+O\left(\frac{1}{z^{N+1}}\right)\right\}
\end{aligned}
$$

we see that there are constants $C(\nu, k)$ such that

$$
\frac{e^{-z}}{z^{1 / 2} z^{\nu}}=\left(\frac{2}{\pi}\right)^{1 / 2} \frac{K_{\nu}(z)}{z^{\nu}}+\sum_{k=1}^{N} \frac{C(\nu, k) e^{-z}}{z^{1 / 2} z^{\nu+k}}+\frac{e^{-z}}{z^{1 / 2}} O\left(\frac{1}{z^{v+N+1}}\right)
$$

and similarly,

$$
\begin{aligned}
& \frac{e^{-z}}{z^{1 / 2} z^{v+1}}=\left(\frac{2}{\pi}\right)^{1 / 2} \frac{K_{v+1}(z)}{z^{v+1}}+\sum_{k=1}^{N-1} \frac{C(\nu+1, k) e^{-z}}{z^{1 / 2} z^{v+1+k}}+\frac{e^{-z}}{z^{1 / 2}} O\left(\frac{1}{z^{v+N+1}}\right) \\
& \vdots \\
& \frac{e^{-z}}{z^{1 / 2} z^{v+N}}=\left(\frac{2}{\pi}\right)^{1 / 2} \frac{K_{v+N}(z)}{z^{v+N}}+\frac{e^{-z}}{z^{1 / 2}} O\left(\frac{1}{z^{v+N+1}}\right) .
\end{aligned}
$$

Now replace the first term of the sum $\sum_{k=1}^{N}$ in (2.8) by the expression given by the first of the equations (2.9). Next apply the second of the equations (2.9) and so forth until the sum in (2.8) has been reduced to one of the form $\sum_{k=1}^{N} C_{k} K_{v+k}(z) / z^{v+k}$. Taking $\nu=2 a-\frac{1}{2}$ the existence of constants $C_{k}(a)$ is evident. The uniqueness of the constants follows by the usual argument for uniqueness of the coefficients in an asymptotic expansion. For future reference we observe that $C_{0}(a)=(2 / \pi)^{1 / 2}$.

To obtain the expansion for $W_{t, a}(r)$ put $z=i t\left(1+x^{2}\right)^{1 / 2}$ in (2.6) and multiply both sides by $(i t)^{2 a} r^{-\mu} x^{\mu+1} J_{\mu}(r x)$. Then integrate over $0 \leqq x<\infty$. Using (2.3) and (2.5) the resulting expression reduces to

$$
W_{t, a}(\boldsymbol{r})=\sum_{k=0}^{N} C_{k}(a)(i t)^{-k} \psi_{2 a+k}(\boldsymbol{r}, t)+R_{N, a}(\boldsymbol{r}, t)
$$

where

$$
\begin{aligned}
\psi_{s}(r, t) & =(i t)^{1-s}\left(\left(r^{2}-t^{2}\right)^{1 / 2}\right)^{s-(n+1) / 2} K_{s-(n+1) / 2}\left(\left(r^{2}-t^{2}\right)^{1 / 2}\right) \\
R_{N, a}(r, t) & =\int_{0}^{\infty} \frac{x^{\mu+1}}{r^{\mu}} J_{\mu}(r x) \frac{\exp \left[-i t\left(1+x^{2}\right)^{1 / 2}\right] B_{N, a}\left(i t\left(1+x^{2}\right)^{1 / 2}\right)}{(i t)^{N+1}\left(\left(1+x^{2}\right)^{1 / 2}\right)^{2 a+N+1}} d x
\end{aligned}
$$


In deriving (2.10) we have assumed $4 a>n+1$ in order that the integrands of (2.3) and (2.5) be absolutely integrable.

THEOREM 2. Let $4 a>n-1$ and take the expansion (2.10) as the definition of $W_{t, a}(\boldsymbol{r})$ for $t>0$. Then

$$
\hat{W}_{t, a}(y)=\left(1+y^{2}\right)^{-a} \exp \left[-i t\left(1+y^{2}\right)^{1 / 2}\right] .
$$

REMARK. By the way in which we arrived at $(2.10)$ the theorem is clearly true when $4 a>n+1$.

Proof. We first check that it is all right to use (2.10) as a definition of $W_{t, a}$. If $4 a>n-1$ the integrand of (2.12) is absolutely integrable. Hence all terms on the right-hand side of (2.10) are well defined. That the choice of the nonnegative integer $N$ does not affect the definition of $W_{t, a}$ is clear when $4 a>n+1$. That this remains true when $4 a>n-1$ can be seen by using analytic continuation for $\operatorname{Re} a>(n-1) / 4$.

Now we check that the Fourier transform of $W_{t, a}$ exists when $4 a>n-1$. Taking $N=0$ in (2.10) gives

$$
W_{t, a}(\boldsymbol{r})=(2 / \pi)^{1 / 2} \psi_{2 a}(\boldsymbol{r}, t)+R_{0, a}(\boldsymbol{r}, t) .
$$

By applying (2.2) to (2.12) we see that $R_{0, a}(r, t)$ is the inverse Fourier transform of the $L_{2}\left(R^{n}\right)$ function

$$
G_{t, a}(x)=\frac{\exp \left[-i t\left(1+x^{2}\right)^{1 / 2}\right] B_{0, a}\left(i t\left(1+x^{2}\right)^{1 / 2}\right)}{i t\left(\left(1+x^{2}\right)^{1 / 2}\right)^{2 a+1}} .
$$

From Theorem 3 (below) we see that $\psi_{2 a}(\cdot, t)$ is in $L_{1}\left(R^{n}\right)$ iff $4 a>n-1$. This shows $W_{t, a}$ is a sum of an $L_{1}$ and an $L_{2}$ function and hence $\hat{W}_{t, a}$ exists.

By Plancherel's theorem, $\left(R_{0, a}(\cdot, t)\right)^{\wedge}(x)=G_{t, a}(x)$. Thus the conclusion of the theorem is equivalent to the statement that

$$
(2 / \pi)^{1 / 2}\left(\psi_{2 a}(\cdot, t)\right)^{\wedge}(x)=\left(1+x^{2}\right)^{-a} \exp \left[-i t\left(1+x^{2}\right)^{1 / 2}\right]-G_{t, a}(x) .
$$

We know (2.13) holds when $4 a>n+1$ because we know the theorem is true then. That (2.13) remains true for $4 a>n-1$ follows by analytic continuation.

3. Analysis of the terms in the expansion. In this section we collect some facts about the functions $\psi_{2 a+k}(r, t)$ involved in the expansion (2.10).

TheOREM 3. For every $t>0, \psi_{s}(\cdot, t)$ is in $L_{p}\left(R^{n}\right)$ if and only if $-1 / p<s-(n+1) / 2$ and in that case

$$
\begin{aligned}
\left\|\psi_{s}(\cdot, t)\right\|_{p} & \simeq t^{n / p} t^{-n / 2} & & \text { when } 1-s-2 / p<-n / 2, \\
& \simeq(\log t)^{1 / p} t^{n / p} t^{-n / 2} & & \text { when } 1-s-2 / p=-n / 2, \\
& \simeq t^{1-s} t^{(n-2) / p} & & \text { when } 1-s-2 / p>-n / 2,
\end{aligned}
$$

where $f(t) \simeq g(t)$ means that $f(t) / g(t)=O(1)$ and $g(t) / f(t)=O(1)$ as $t \rightarrow \infty$. 
For $p=\infty$ we have the slightly strengthened version:

THEOREM $3^{\prime}$. If $w=s-(n+1) / 2 \leqq 0$ then $\psi_{s}(r, t)$ is unbounded along the cone $r=t$. If $w>0$ then $\psi_{s}(\boldsymbol{r}, t)$ is continuous on $\left\{(\boldsymbol{r}, t): \boldsymbol{r} \in R^{n}\right.$ and $\left.t>0\right\}$ and

$$
\begin{aligned}
\left\|\psi_{s}(\cdot, t)\right\|_{\infty} & \sim(\pi / 2)^{1 / 2} t^{-n / 2} \quad \text { when } 1-s \leqq-n / 2, \\
& \sim 2^{w-1} \Gamma(w) t^{1-s} \quad \text { when } 1-s \geqq-n / 2,
\end{aligned}
$$

where $f(t) \sim g(t)$ means $f(t) / g(t) \rightarrow 1$ as $t \rightarrow \infty$.

Proof of Theorem 3'. From the definition (2.11) we see that the continuity of $\psi_{s}(r, t)$ for $t>0$ is in doubt only on the cone $r=t$ where $u=\left(r^{2}-t^{2}\right)^{1 / 2}$ changes from the positive real axis to the positive imaginary axis. By looking at first terms of power series for $K_{w}(z)$ one finds that

$$
\begin{array}{ll}
\lim _{x \rightarrow 0+} x^{w} K_{w}(x)=2^{w-1} \Gamma(w)=\lim _{x \rightarrow 0+}(i x)^{w} K_{w}(i x) & \text { if } w>0, \\
\lim _{x \rightarrow 0+} x^{w} K_{w}(x)=\infty=\lim _{x \rightarrow 0+}|i x|^{w}\left|K_{w}(i x)\right| & \text { if } w \leqq 0 .
\end{array}
$$

The first part of the theorem follows easily from these equations. To complete the proof we note that

$$
\left\|\psi_{s}(\cdot, t)\right\|_{\infty}=t^{1-s} \max \left(A_{w}(t), B_{w}(t)\right)
$$

where $w=s-(n+1) / 2$ and

$$
\begin{aligned}
& A_{w}(t)=\sup _{r>t}\left|u^{w} K_{w}(u)\right|=\sup _{x>0} x^{w} K_{w}(x), \\
& B_{w}(t)=\sup _{0<r<t}\left|u^{w} K_{w}(u)\right|=\sup _{0<x<t}\left|x^{w} K_{w}(i x)\right| .
\end{aligned}
$$

When $1-s \leqq-n / 2$ we have $\frac{1}{2} \leqq w$ and the theorem follows from Lemmas 3.1 and 3.3 below. When $1-s \geqq-n / 2$ we have $w \leqq \frac{1}{2}$ and the theorem follows from Lemmas 3.1 and 3.2 .

LEMMA 3.1. If $w>0$ then $A_{w}(t)=2^{w-1} \Gamma(w)$.

Proof. $K_{w}(x)$ is positive for $x>0$ and $x^{-1}(d / d x) x^{w} K_{w}(x)=-x^{w-1} K_{w-1}(x) \leqq 0$. Hence

$$
\sup _{x>0} x^{w} K_{w}(x)=\lim _{x \rightarrow 0+} x^{w} K_{w}(x)=2^{w-1} \Gamma(w) \quad \text { by (3.1a). }
$$

LEMMA 3.2. If $0<w \leqq \frac{1}{2}$ then $B_{w}(t)=2^{w-1} \Gamma(w)$.

Proof. For $w=\frac{1}{2}$ the result follows from $z^{1 / 2} K_{1 / 2}(z)=(\pi / 2)^{1 / 2} e^{-z}$. For $0<w<\frac{1}{2}$ we have from $\$ 6.15$ of Watson [8] that

$$
z^{w} K_{w}(z)=\frac{2^{w} \Gamma\left(\frac{1}{2}\right)}{\Gamma\left(\frac{1}{2}-w\right)} \int_{0}^{\infty} \frac{e^{-z \cosh \theta}}{\sinh ^{2 w} \theta} d \theta
$$


when $\operatorname{Re} z \geqq 0$. Since $\left|e^{-i x \cosh \theta}\right| \leqq 1$ this implies

$$
\left|(i x)^{w} K_{w}(i x)\right| \leqq \frac{2^{w} \Gamma\left(\frac{1}{2}\right)}{\Gamma\left(\frac{1}{2}-w\right)} \int_{0}^{\infty} \frac{1}{\sinh ^{2 w} \theta} d \theta=\lim _{x \rightarrow 0+} x^{w} K_{w}(x) .
$$

Now apply (3.1) to obtain the lemma.

LEMMA 3.3. If $w \geqq \frac{1}{2}$ then $B_{w}(t) \sim(\pi / 2)^{1 / 2} t^{w-1 / 2}$ as $t \rightarrow \infty$.

Proof. The case $w=\frac{1}{2}$ is immediate from Lemma 3.2 so we assume $w>\frac{1}{2}$. Let $0<\varepsilon<1$. Using (2.7) take $x_{0}$ such that $(1-\varepsilon)(\pi / 2 x)^{1 / 2}<\left|K_{w}(i x)\right|<(1+\varepsilon)(\pi / 2 x)^{1 / 2}$ when $x>x_{0}$. Next, using $w-\frac{1}{2}>0$ take $x_{1}$ such that

$$
\sup _{0<x<x_{0}}\left|x^{w} K_{w}(i x)\right|<(1-\varepsilon)(\pi / 2)^{1 / 2} x_{1}^{w-1 / 2} .
$$

If $t>x_{0}$ then

$$
(1-\varepsilon)(\pi / 2)^{1 / 2} t^{w-1 / 2}<\left|t^{w} K_{w}(i t)\right| \leqq B_{w}(t) .
$$

If $t>\max \left(x_{0}, x_{1}\right)$ we see from (3.2) and the choice of $x_{1}$ that

$$
\sup _{0<x<x_{0}}\left|x^{w} K_{w}(i x)\right|<B_{w}(t)
$$

and hence

$$
B_{w}(t)=\sup _{x_{0}<x<t}\left|x^{w} K_{w}(i x)\right| \leqq \sup _{x_{0}<x<t} x^{w}(1+\varepsilon)(\pi / 2 x)^{1 / 2}=(1+\varepsilon)(\pi / 2)^{1 / 2} t^{w-1 / 2} .
$$

Combining this with (3.2) we have $1-\varepsilon<B_{w}(t) /(\pi / 2)^{1 / 2} t^{w-1 / 2}<1+\varepsilon$ for $t>$ $\max \left(x_{0}, x_{1}\right)$ and the lemma follows.

Proof of Theorem 3. Let $w=s-(n+1) / 2$ and define

$$
I(t)=\int_{0}^{\infty}\left|\left(r^{2}-t^{2}\right)^{1 / 2}\right| w p\left|K_{w}\left(\left(r^{2}-t^{2}\right)^{1 / 2}\right)\right|^{p} r^{n-1} d r .
$$

Then $\left\|\psi_{s}(\cdot, t)\right\|_{p}=t^{1-s}\left(\omega_{n} I(t)\right)^{1 / p}$ where $\omega_{n}$ is the area of the unit sphere in $R^{n}$. To prove Theorem 3 it suffices to establish

LEMMA 3.4. For every $t>0, I(t)$ is finite iff $w p>-1$ and in that case

$$
\begin{aligned}
I(t) & \simeq t^{n+p(w-1 / 2)} & & \text { when } p\left(w-\frac{1}{2}\right)>-2, \\
& \simeq t^{n-2} \log t & & \text { when } p\left(w-\frac{1}{2}\right)=-2, \\
& \simeq t^{n-2} & & \text { when } p\left(w-\frac{1}{2}\right)<-2 .
\end{aligned}
$$

REMARK. In the proof we make use of the fact that $f(t) \simeq g(t)$ if there exists a constant $c \neq 0$ such that $c^{-1} f(t) \leqq g(t) \leqq c f(t)$ for all large $t$.

Proof. We break the range of integration for $I(t)$ into the intervals $[0, t]$ and $[t, \infty)$. First let

$$
\begin{aligned}
I_{1}(t) & =\int_{t}^{\infty}\left|\left(r^{2}-t^{2}\right)^{1 / 2}\right|^{w p}\left|K_{w}\left(\left(r^{2}-t^{2}\right)^{1 / 2}\right)\right|^{p} r^{n-1} d r \\
& =\int_{0}^{\infty} y^{w p}\left|K_{w}(y)\right|^{p}\left(\left(t^{2}+y^{2}\right)^{1 / 2}\right)^{n-2} y d y
\end{aligned}
$$


where we have made the change of variable $y=\left(r^{2}-t^{2}\right)^{1 / 2}$. Next apply the change of variable $y=\left(t^{2}-r^{2}\right)^{1 / 2}$ to the remaining interval $0 \leqq r \leqq t$ to obtain

$$
I(t)=I_{1}(t)+\int_{0}^{t}|i y|^{w p}\left|K_{w}(i y)\right|^{p}\left(\left(t^{2}-y^{2}\right)^{1 / 2}\right)^{n-2} y d y .
$$

Since $K_{w}(y)$ decreases exponentially as $y \rightarrow \infty$, the finiteness of $I_{1}(t)$ depends only on the behavior of the integrand at $y=0$. Using the fact that at $y=0, K_{w}(y)$ and $\left|K_{w}(i y)\right|$ behave like $y^{-|w|}$ (or $|\log y|$ if $w=0$ ) it is easily checked that $I(t)$ is finite iff $w p>-1$.

Now use (2.7) to choose $b=b(w)$ such that $y \geqq b$ implies $(\pi / 4 y)^{1 / 2}<\left|K_{w}(i y)\right|<$ $(\pi / y)^{1 / 2}$. For $t>b$ split the integral appearing in (3.3) into the two parts

$$
\begin{aligned}
& I_{2}(t)=\int_{0}^{b} y^{w p}\left|K_{w}(i y)\right|^{p}\left(\left(t^{2}-y^{2}\right)^{1 / 2}\right)^{n-2} y d y, \\
& I_{3}(t)=\int_{b}^{t} y^{w p}\left|K_{w}(i y)\right|^{p}\left(\left(t^{2}-y^{2}\right)^{1 / 2}\right)^{n-2} y d y .
\end{aligned}
$$

By the choice of $b$ and the change of variable $y=t \sin \theta$ we have

$$
I_{3}(t) \simeq \int_{b}^{t} y^{q}\left(\left(t^{2}-y^{2}\right)^{1 / 2}\right)^{n-2} d y=t^{q+n-1} \int_{\theta_{t}}^{\pi / 2}(\sin \theta)^{q}(\cos \theta)^{n-1} d \theta
$$

where $q=w p-p / 2+1$ and $\theta_{t}=\sin ^{-1}(b / t)$.

Let $J(t)=\int_{\theta_{t}}^{\pi / 2}(\sin \theta)^{q}(\cos \theta)^{n-1} d \theta$. If $q>-1$ then

$$
\lim _{t \rightarrow \infty} J(t)=\int_{0}^{\pi / 2}(\sin \theta)^{q}(\cos \theta)^{n-1} d \theta .
$$

For $q \leqq-1$ we note that

$$
\begin{array}{rlrl}
J(t) \simeq \int_{\theta_{t}}^{\pi / 2}(\sin \theta)^{q} d \theta \simeq \int_{\theta_{t}}^{\pi / 2} \theta^{q} d \theta & \simeq-\log \theta_{t} & \text { if } q=-1, \\
& \simeq \theta_{t}^{q+1} \quad \text { if } q<-1 .
\end{array}
$$

Since $b / t \leqq \theta_{t} \leqq \pi b / 2 t$ and $\log b-\log t \leqq \log \theta_{t}<\log (\pi b / 2)-\log t$ we have $\theta_{t}^{q+1} \simeq t^{-q-1}$ and $-\log \theta_{t} \simeq \log t$. Thus

so by (3.4)

$$
\begin{aligned}
J(t) & \simeq 1 & & \text { if } q>-1 \\
& \simeq \log t & & \text { if } q=-1 \\
& \simeq t^{-q-1} & & \text { if } q<-1
\end{aligned}
$$

$$
\begin{aligned}
I_{3}(t) & \simeq t^{n+p(w-1 / 2)} & & \text { if } q=p w-p / 2+1>-1 \\
& \simeq t^{n-2} \log t & & \text { if } q=p w-p / 2+1=-1 \\
& \simeq t^{n-2} & & \text { if } q=p w-p / 2+1<-1
\end{aligned}
$$

To complete the proof of the lemma it suffices to show

$$
I_{1}(t)+I_{2}(t)=O\left(t^{n-2}\right)
$$


because then $I_{3}(t) \leqq I(t) \leqq I_{3}(t)+O\left(t^{n-2}\right)$ so that the lemma follows from (3.5). To prove (3.6) when $n=1$ apply $1 /\left(t^{2}+y^{2}\right)^{1 / 2} \leqq 1 / t$ to the integral for $I_{1}(t)$ and apply $1 /\left(t^{2}-y^{2}\right)^{1 / 2}=1 / t\left(1-(y / t)^{2}\right)^{1 / 2} \leqq 1 / t(3 / 4)^{1 / 2}, t>2 b \geqq 2 y$, to the integral for $I_{2}(t)$. When $n=2$ the integrals for $I_{1}(t)$ and $I_{2}(t)$ do not depend on $t$ so (3.6) is obvious. When $n \geqq 3$ use $\left(t^{2}-y^{2}\right)^{1 / 2} \leqq\left(t^{2}+y^{2}\right)^{1 / 2} \leqq t\left(1+y^{2}\right)^{1 / 2}, t \geqq 1$.

4. Estimates on the remainder. From equations (2.2) and (2.12) we have

$$
R_{N, a}(r, t)=(2 \pi)^{-n / 2} \int_{R^{n}} \frac{e^{i x \cdot r} \exp \left[-i t\left(1+x^{2}\right)^{1 / 2}\right] B_{N, a}\left(i t\left(1+x^{2}\right)^{1 / 2}\right)}{(i t)^{N+1}\left(\left(1+x^{2}\right)^{1 / 2}\right)^{2 a+N+1}} d x_{1} \cdots d x_{n}
$$

By Lemma $2.1 B_{N, a}\left(i t\left(1+x^{2}\right)^{1 / 2}\right)$ is continuous for $t>0$ and $\left|B_{N, a}\left(i t\left(1+x^{2}\right)^{1 / 2}\right)\right| \leqq$ $\left|B_{N, a}\right|_{\varepsilon}$ for $t \geqq \varepsilon>0$. Thus if $2 a+N+1>n$ the integrand of (4.1) will be in $L_{1}\left(R^{n}\right)$ and $R_{N, a}(r, t)$ will be continuous on $\left\{(\boldsymbol{r}, t): \boldsymbol{r} \in R^{n}\right.$ and $\left.t>0\right\}$. Furthermore, we then have the bound

$$
\left|R_{N}(\boldsymbol{r}, t)\right| \leqq t^{-N-1}\left|B_{N, a}\right|_{\varepsilon} \omega_{n}(2 \pi)^{-n / 2} \int_{0}^{\infty}\left(1+x^{2}\right)^{-(2 a+N+1) / 2} x^{n-1} d x
$$

for $t \geqq \varepsilon$, where $\omega_{n}$ is the area of the unit sphere in $R^{n}$. By Parseval's equality we also see from (4.1) that $R_{N, a}(\cdot, t)$ is in $L_{2}\left(R^{n}\right)$ if $2 a+N+1>n / 2$ and in fact

$$
\left\|R_{N}(\cdot, t)\right\|_{2}=O\left(t^{-N-1}\right) .
$$

THEOREM 4. If $4 a>n-1$ we have for each integer $N \geqq 0$ that

(i) $R_{N, a}(r, t)$ is continuous on $\left\{(\boldsymbol{r}, t): \boldsymbol{r} \in R^{n}\right.$ and $\left.t>0\right\}$,

(ii) $\left\|R_{N, a}(\cdot, t)\right\|_{2}=O\left(t^{-(N+1)}\right)$ as $t \rightarrow \infty$,

(iii) $\left\|R_{N, a}(\cdot, t)\right\|_{\infty}=O\left(t^{-(m+N+1)}\right)$ as $t \rightarrow \infty$, where $m=\min (n / 2,2 a+N)$.

REMARK. Note that $4 a>n-1$ implies $2 a+N>n / 2+N-\frac{1}{2}$ so that $m=n / 2$ unless $N=0$ and $n>4 a$.

Proof. Part (ii) follows from (4.3) because $4 a>n-1$ implies $2 a+N+1>n / 2$. From the expansion (2.10) we have

$$
R_{N, a}(\boldsymbol{r}, t)=\sum_{k=N+1}^{N+K} C_{k}(a)(i t)^{-k} \psi_{2 a+k}(\boldsymbol{r}, t)+R_{N+K, a}(\boldsymbol{r}, t) .
$$

To finish the proof it suffices to show that each term $f(\boldsymbol{r}, t)$ on the right is continuous for $t>0$ and satisfies $\|f(\cdot, t)\|_{\infty}=O\left(t^{-(m+N+1)}\right)$.

Inequality (4.2) and the discussion preceding it show that if $K$ is large enough, the term $R_{N+K, a}(r, t)$ meets both requirements. Theorem $3^{\prime}$ guarantees that $\psi_{2 a+k}(\boldsymbol{r}, t)$ will be continuous for $t>0$ provided $2 a+k>(n+1) / 2$. This condition is automatically satisfied because $k \geqq N+1 \geqq 1$ and $4 a>n-1$. Thus it only remains to show that $\left\|\psi_{2 a+k}(\cdot, t)\right\|_{\infty}=O\left(t^{-m}\right), k \geqq N+1$.

Case $m=n / 2$. In this case $2 a+k \geqq 2 a+N+1 \geqq n / 2+1$. Hence by Theorem $3^{\prime}$

$$
\left\|\psi_{2 a+k}(\cdot, t)\right\|_{\infty}=O\left(t^{-n / 2}\right) .
$$


Case $m<n / 2$. In this case $n>4 a>n-1, N=0$ and $m=2 a$. Thus we have $n / 2+1>2 a+1>(n+1) / 2$ so that by Theorem $3^{\prime}\left\|\psi_{2 a+1}(\cdot, t)\right\|_{\infty}=O\left(t^{-2 a}\right)$. For $k \geqq 2$ we have $2 a+k>n / 2+1$ so that (4.5) holds.

5. Conclusion. Writing equation (2.11) with $N=0$ and noting that $C_{0}(a)$ $=(2 / \pi)^{1 / 2}$ we have

$$
W_{t, a}(r)=(2 / \pi)^{1 / 2} \psi_{2 a}(r, t)+R_{0, a}(r, t), \quad t>0 .
$$

Applying Theorems $3,3^{\prime}$ and 4 to this we obtain the following corollaries.

Corollary 5.1. Fix $a>(n-1) / 4$ and $2 \leqq p<\infty$. Then for every $t \neq 0, W_{t, a}$ is in $L_{p}\left(R^{n}\right)$ iff $2 a-(n+1) / 2>-1 / p$ in which case $\left\|W_{t, a}\right\|_{p} \sim(2 / \pi)^{1 / 2}\left\|\psi_{2 a}(\cdot, t)\right\|_{p}$ so that

$$
\begin{aligned}
\left\|W_{t, a}\right\|_{p} & \simeq t^{n / p} t^{-n / 2} & & \text { if }(p, a) \in S_{1}, \\
& \simeq(\log t)^{1 / p} t^{n / p} t^{-n / 2} & & \text { if }(p, a) \in S_{2}, \\
& \simeq t^{1-2 a} t^{(n-2) / p} & & \text { if }(p, a) \in S_{3},
\end{aligned}
$$

where

$$
\begin{aligned}
& S_{1}=\{(p, a): p(2 a-(n+2) / 2)>-2 \text { and } p \geqq 2\}, \\
& S_{2}=\{(p, a): p(2 a-(n+2) / 2)=-2 \text { and } p>2\}, \\
& S_{3}=\{(p, a): p(2 a-(n+2) / 2)<-2 \text { and } p(2 a-(n+1) / 2)>-1\}
\end{aligned}
$$

are the regions shown in Figure 1.

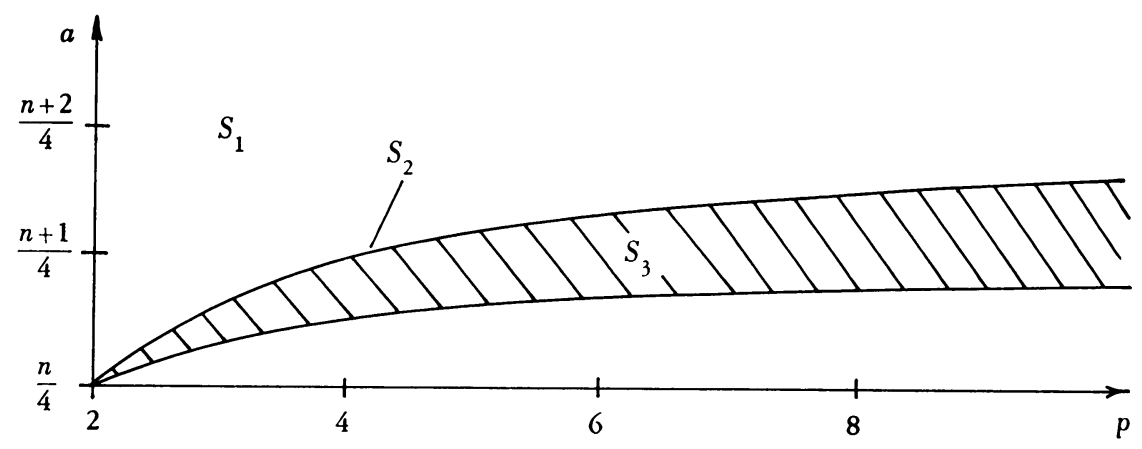

FIGURE 1

REMARK. If $n=2$ the decay rate of $\left\|W_{t, a}\right\|_{p}$ in the region $S_{3}$ does not depend on $p$ and if $n=1$ faster decay rates in the region $S_{3}$ are obtained by taking smaller values of $p$ keeping $a$ fixed. With these exceptions, one has the rule that for fixed values of $a$, increasing $p$ increases the decay rate of $\left\|W_{t, a}\right\|_{p}$.

COROLlaRY 5.2. If $n+1 \geqq 4 a>n-1$ then $W_{t, a}(r)$ is unbounded along the cone $r=t>0$. If $4 a>n+1$ then

(i) $W_{t, a}(\boldsymbol{r})$ is continuous on $\left\{(\boldsymbol{r}, t) \in R^{n+1}:(\boldsymbol{r}, t) \neq(\mathbf{0}, 0)\right\}$, 
(ii) $W_{t, a}$ is in $L_{\infty}\left(R^{n}\right)$ for $t>0$ and

$$
\begin{aligned}
\left\|W_{t, a}\right\|_{\infty} & \sim t^{-n / 2} & & \text { if } 4 a \geqq n+2, \\
& \sim c_{n, a} t^{1-2 a} & & \text { if } 4 a \leqq n+2,
\end{aligned}
$$

where $c_{n, a}=2^{2 a-1-n / 2} \Gamma(2 a-(n+1) / 2) / \Gamma\left(\frac{1}{2}\right)$.

RemarK. Using Equation (2.3) with [1, Equation 20, page 24, volume 2] one has

$$
W_{0, a}(\boldsymbol{r})=\frac{r^{a-n / 2} K_{a-n / 2}(r)}{2^{a-1} \Gamma(a)}
$$

which is unbounded at $r=0$ if $2 a \leqq n$. Thus it it necessary to exclude the origin in part (i) unless $2 a>n$. If $2 a>n$ then $\left(1+x^{2}\right)^{-a}$ is in $L_{1}\left(R^{n}\right)$ so it is easily seen from equation (1.1) that $W_{t, a}(\boldsymbol{r})$ is continuous for all $(\boldsymbol{r}, t)$ in $R^{n+1}$.

Proof of Corollary 5.1. Using $p \geqq 2$ we have

$$
\begin{aligned}
\left\|R_{0, a}(\cdot, t)\right\|_{p}^{p} & =\int_{R^{n}}\left|R_{0, a}(x, t)\right|^{2}\left|R_{0, a}(x, t)\right|^{p-2} d x_{1} \cdots d x_{n} \\
& \leqq\left\|R_{0, a}(\cdot, t)\right\|_{\infty}^{p-2}\left\|R_{0, a}(\cdot, t)\right\|_{2}^{2}
\end{aligned}
$$

Since $a>(n-1) / 4$ this is finite by Theorem 4. Therefore it follows from (5.1) that $W_{t, a}$ is in $L_{p}\left(R^{n}\right)$ iff $\psi_{2 a}(\cdot, t)$ is in $L_{p}\left(R^{n}\right)$. By Theorem 3 this is so iff $2 a-(n+1) / 2$ $>-1 / p$.

Now assume $2 a-(n+1) / 2>-1 / p$. Since $p \geqq 2$ this implies $2 a>n / 2$ so that by Theorem $4\left\|R_{0, a}(\cdot, t)\right\|_{\infty}=O\left(t^{-(n / 2+1)}\right)$. Also by Theorem 4 we have $\left\|R_{0, a}(\cdot, t)\right\|_{2}$ $=O\left(t^{-1}\right)$ and hence from (5.3) it follows that $\left\|R_{0, a}(\cdot, t)\right\|_{p}=O\left(t^{-h}\right)$ where $h=(n / 2+1)(1-2 / p)+2 / p=n / 2+1-n / p$. Using this with Theorem 3 it is straightforward to complete the proof.

Proof of Corollary 5.2. Only part (i) requires explanation. Since $W_{-t, a}(\boldsymbol{r})$ equals the complex conjugate of $W_{t, a}(\boldsymbol{r})$, it follows from Theorems $3^{\prime}$ and 4 that $W_{t, a}(\boldsymbol{r})$ is continuous on $\left\{(\boldsymbol{r}, t): r \in R^{n}\right.$ and $\left.t \neq 0\right\}$. By equation (2.3) and Lebesgue's dominated convergence theorem, $W_{t, a}(\boldsymbol{r})$ is continuous on $\{(\boldsymbol{r}, t): \boldsymbol{r} \neq \mathbf{0}$ and $t \in R\}$. Combining these results gives part (i).

\section{REFERENCES}

1. H. Bateman Manuscript Project, Tables of integral transforms, A. Erdelyi (editor), McGraw-Hill, New York, 1954.

2. S. Bochner and K. Chandrasekharan, Fourier transforms, Ann. of Math. Studies, no. 19, Princeton Univ. Press, Princeton, N. J., 1949. MR 11, 173.

3. A. R. Brodsky, Asymptotic decay of solutions to the relativistic wave equation and the existence of scattering for certain non-linear hyperbolic equations, Doctoral Thesis, Department of Mathematics, Massachusetts Institute of Technology, Cambridge, Mass., 1964.

4. S. Nelson, Asymptotic behavior of certain (quasi-) fundamental solutions to the KleinGordon equation, Doctoral Thesis, Department of Mathematics, Massachusetts Institute of Technology, Cambridge, Mass., 1966.

5. - $L^{2}$ asymptotes for the Klein-Gordon equation, Proc. Amer. Math. Soc. (to appear). 
6. I. E. Segal, Quantization and dispersion for nonlinear relativistic equations, Proc. Conference Math. Theory of Elementary Particles (Dedham, Mass., 1965), M.I.T. Press, Cambridge, Mass., 1966. MR 36 \#542.

7. _- Dispersion for nonlinear relativistic equations. II, Ann. Sci. École Norm. Sup. (4) 1 (1968), 459-497.

8. G. N. Watson, Theory of Bessel functions, Cambridge Univ. Press, New York, 1922.

9. W. Littman, Fourier transforms of surface-carried measures and differentiability of surface averages, Bull. Amer. Math. Soc. 69 (1963), 766-770. MR 27 \#5086.

Iowa State University,

Ames, Iowa 50010 